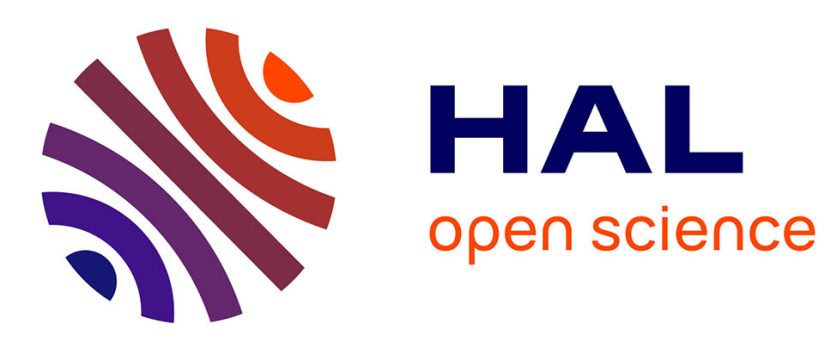

\title{
'Diese meine Sprache, die so männlich geworden ist.' Jeckes in Palästina/Israel im Spannungsverhältnis zwischen Sprachen und Geschlecht
}

\author{
Patrick Farges
}

\section{- To cite this version:}

Patrick Farges. 'Diese meine Sprache, die so männlich geworden ist.' Jeckes in Palästina/Israel im Spannungsverhältnis zwischen Sprachen und Geschlecht. L'Homme. Zeitschrift für feministische Geschichtswissenschaft, 2015, Mit Sprachen, 26 (1), pp.63-78. 10.7767/lhomme-2015-0106 . halshs01379695

\section{HAL Id: halshs-01379695 \\ https://shs.hal.science/halshs-01379695}

Submitted on 31 Mar 2020

HAL is a multi-disciplinary open access archive for the deposit and dissemination of scientific research documents, whether they are published or not. The documents may come from teaching and research institutions in France or abroad, or from public or private research centers.
L'archive ouverte pluridisciplinaire HAL, est destinée au dépôt et à la diffusion de documents scientifiques de niveau recherche, publiés ou non, émanant des établissements d'enseignement et de recherche français ou étrangers, des laboratoires publics ou privés. 


\section{GENDER}

OPEN

REPOSITORIUM

Repositorium für die Geschlechterforschung

\section{"Diese meine Sprache, die so männlich geworden ist." : Jeckes in Palästina/lsrael im Spannungsverhältnis zwischen Sprachen und Geschlecht}

Farges, Patrick

2015

https://doi.org/10.25595/1169

Veröffentlichungsversion / published version

Zeitschriftenartikel / journal article

\section{Empfohlene Zitierung / Suggested Citation:}

Farges, Patrick: "Diese meine Sprache, die so männlich geworden ist." : Jeckes in Palästina/lsrael im Spannungsverhältnis zwischen Sprachen und Geschlecht, in: L' homme : Zeitschrift für feministische Geschichtswissenschaft, Jg. 26 (2015) Nr. 1, 63-78. DOI: https://doi.org/10.25595/1169.

Erstmalig hier erschienen / Initial publication here: https://doi.org/10.7767/lhomme-2015-0106

Nutzungsbedingungen:

Dieser Text wird unter einer CC BY 4.0 Lizenz (Namensnennung) zur Verfügung gestellt. Nähere Auskünfte zu dieser Lizenz finden Sie hier:

https://creativecommons.org/licenses/by/4.0/deed.de
Terms of use:

This document is made available under a CC BY 4.0 License (Attribution). For more information see:

https://creativecommons.org/licenses/by/4.0/deed.en 


\title{
"Diese meine Sprache, die so männlich geworden ist." Jeckes in Palästina/Israel im Spannungsverhältnis zwischen Sprachen und Geschlecht
}

\author{
Patrick Farges
}

\section{Sprachkontakt, Sprachbiographie, Geschlecht}

In den 1930er Jahren emigrierten deutsche Juden und Jüdinnen nach Palästina ${ }^{1}$ im Rahmen der in der zionistischen Geschichtsschreibung so bezeichneten „Fünften Alija“. Im israelischen Sprachgebrauch werden sie "Jeckes“ genannt. Die Etymologie des Begriffs ist zwar umstritten, doch eine gängige Deutung weist direkt auf Sprach- und Kulturkontakte hin: Die jiddischsprachigen Juden des Jischuw ${ }^{2}$ hätten die Neuankömmlinge aus deutschen Großstädten „Jeckes“ genannt. Der Begriff ist mit Konnotationen der Andersartigkeit, einer gewissen Naivität und Humorlosigkeit, aber auch mit Gewissenhaftigkeit, Verlässlichkeit und Anständigkeit verbunden. Der urban legend nach seien die Jeckes unter den EinwohnerInnen Palästinas/Israels an ihren besonders starren Umgangsformen erkennbar gewesen, an ihrem „etwas altmodischen, stehengebliebenen, ja versteinerten Deutsch“, ihrer Vorliebe für Kultur und Musik und vor allem ihrer für das orientalische Klima völlig ungeeigneten Kleidung: Die Männer hätten - so die Legende - das bis oben zugeknöpfte Jackett - Jiddisch: Jecke - nach Berliner Art getragen. Dieses altmodische Kleidungsstück fungiert als Symbol einer vergangenen Grandezza, die reichlich verspottet wird. Hier nehmen die Ostjuden eine historische Revanche an den ,assimilierten' Westjuden.

I Der Begriff „Palästina“ bezeichnet hier die westjordanische Region, die zwischen 1922 und 1948 unter britischem Mandat stand. Der Begriff bezeichnet also das ,vorstaatliche“ Israel. Die Emigration von deutschen Juden und Jüdinnen nach Palästina hatte zwar schon vor 1933 angefangen, doch vor der „Fünften Alija“ waren nur wenige deutschsprachige Juden (etwa 2.000) Mitglied der jüdischen Bevölkerung Palästinas. In diesem Artikel geht es v. a. um die mit dem Nationalsozialismus einsetzende Emigrationswelle. Zur Präsenz von deutschen Juden in Palästina vor 1933 vgl. Etan Bloom, Arthur Ruppin and the Production of the Modern Hebrew Culture, Dissertation, Universität Tel Aviv 2008.

2 Die jüdische Bevölkerung Palästinas.

3 Anne Betten u. Miryam Du-nour, Wir sind die Letzten. Fragt uns aus. Gespräche mit den Emigranten der dreißiger Jahre in Israel, Gerlingen 1995 (Neuauflage Gießen 2004), 10. 
Die nach Palästina ausgewanderten Juden und Jüdinnen aus Deutschland und Österreich erfuhren Entortung und Neu-Verortung maßgeblich sprachlich. Davon zeugen - unter anderen narrativen Selbstzeugnissen und Quellen - die Oral-History-Interviews des „Israel-Korpus“, ${ }^{4}$ die in den 1990er Jahren - sechzig Jahre nach der Emigration - in Israel von einer deutsch-israelischen Forschungsgruppe in deutscher Sprache geführt worden waren. Sprachen und Sprachgebräuche sind in sozio-kulturelle Realitäten und emotionale Kontexte eingebettet - sie fungieren als Schnittstellen zwischen kulturellen, sozialen und politischen Machtverhältnissen. Mit diesem Problem befasst sich die Sprachkontaktforschung, die individuelle Phänomene der Mehrsprachigkeit sowie gruppenbezogene Sprachinteraktionen analysiert. ${ }^{5}$ Seit einiger Zeit wendet sich die Sprachkontakt- und Mehrsprachigkeitsforschung verstärkt lebensgeschichtlich-historischen Umständen ${ }^{6}$ sowie der historisch-biographischen Emotionalität von Sprache zu. ${ }^{7}$ Erinnerte Sprachkontakte und erzählte Emotionen werden hier als konstitutive Kernelemente erfahrener Lebenswelten verstanden: Sie bestimmen Bewusstseins-, Denk- und Handlungsschemata mit. In diesem Sinne können retrospektive Oral-History-Interviews als „Sprachbiographien“8 gelten, im Rahmen derer individuelle Erfah-

4 Das „Israel-Korpus (1. Generation)“ besteht aus ca. 150 Oral-History-Interviews mit jüdisch-deutschen EmigrantInnen in Israel, die im Zeitraum 1989 bis 1994 von einer Gruppe deutscher und israelischer Forscherinnen unter der Leitung von Prof. Dr. Anne Betten (Katholische Universität Eichstätt, später Universität Salzburg) und Dr. Myriam Du-nour (Bar-Ilan Universität) geführt wurden. Die Interviewerinnen waren besonders an sprachlichen Aspekten interessiert. Vgl. die Internetpräsentation am Spracharchiv des Instituts für Deutsche Sprache Mannheim (http://dsav-oeff.ids-mannheim.de/DSAv/korpora/ is/is_doku.htm) sowie Anne Betten u. Miryam Du-nour Hg., Sprachbewahrung nach der Emigration Das Deutsch der 20er Jahre in Israel, Teile I und II (Phonai 42 und 45), Tübingen 1995 und 2000; Anne Betten, Die deutsche Sprache bei der 1. und 2. Generation deutschsprachiger Immigranten in Israel, in: Der Sprachdienst, 57, 4-5 (2013): Deutsch in Israel, hg. von Andrea-Eva Ewels, 168-180.

5 Das Pionierwerk in diesem Gebiet stammt vom Sprachwissenschaftler und Jiddischisten Uriel Weinreich (Languages in Contact: Findings and Problems, New York 1953). Zur Sprachkontaktforschung und Kontaktlinguistik vgl. Karl Heinz Wagner u. Wolfgang Wildgen Hg., Studien zum Sprachkontakt (Bremer Linguistisches Kolloquium, Bd. 1), Bremen 1988; Johannes Bechert u. Wolfgang Wildgen, Einführung in die Sprachkontaktforschung, Darmstadt 1991; Hans Goebl, Peter H. Nelde, Zdenek Stary u. Wolfgang Wölck Hg., Kontaktlinguistik. Ein internationales Handbuch zeitgenössischer Forschung, 2 Bände, Berlin 1996-1997; Claudia Maria Riehl, Sprachkontaktforschung. Eine Einführung, Tübingen 2004.

6 Vgl. Rita Franceschini Hg., Sprache und Biographie (Zeitschrift für Literaturwissenschaft und Linguistik - LiLi, 40, 160), Stuttgart/Weimar 2010.

7 Vgl. Monika Schwarz-Friesel, Sprache und Emotion, Tübingen 2013.

8 Zum Begriff der „Sprachbiographie“ vgl. Kirsten Adamzik u. Eva Roos Hg., Biografie linguistiche Biographies langagières - Biografias linguisticas - Sprachbiografien (Bulletin suisse de linguistique appliquée, 76), Neuchâtel 2002; Rita Franceschini u. Johanna Miecznikowski Hg., Leben mit mehreren Sprachen. Vivre avec plusieurs langues. Sprachbiographien - Biographies langagières, Bern 2004. Vgl. besonders Anne Betten, Sprachbiographien deutscher Emigranten. Die ,Jeckes' in Israel zwischen Verlust und Rekonstruktion ihrer kulturellen Identität, in: Arnulf Deppermann Hg., Das Deutsch der Migranten, Berlin/Boston 2013, 145-192. 
rungen, kollektive Erwartungshorizonte - die das Sagbare mitbestimmen - und die daraus resultierenden Identitätskonstruktionen zusammenspielen. Sprachbiographien artikulieren individuelle Erfahrungen, sind aber zugleich auch symptomatisch für kollektiv erlebte Entwicklungen in einer bestimmten historischen Zeit. ${ }^{9}$ Die individuelle narrative Arbeit erlaubt Rückschlüsse etwa auf eingewobene master narratives, auf die retrospektive Anpassung des Erlebten an die biographische Selbstkonstitution, auf Unterschiede zwischen gegenwärtigen und vergangenen Einstellungen sowie auf kollektivhegemoniale Konstruktionen wie etwa Geschlechtervorstellungen. Denn Geschlecht ergibt sich auch performativ über kommunikative und sprachliche Interaktionen: Sprachbiographien und andere Selbstzeugnisse sind somit strategische Aushandlungsund Entfaltungsorte von Geschlechterwelten. ${ }^{10}$

In Palästina/Israel waren (und sind) Sprachkontakte und Sprachhierarchien besonders komplex. Sie dienten zur sozialen, kulturellen und geschlechterspezifischen Positionierung. Im sprachlichen Alltag der Jeckes rangen neben Deutsch und Hebräisch auch Englisch, Arabisch, Jiddisch und andere ,Diaspora'-Sprachen miteinander, die jeweils unterschiedlich wahrgenommen wurden. Es ergibt sich also eine komplexe Sprachsituation, die im Prozess der israelischen Nationsbildung lange unterbeleuchtet blieb, weil das zionistische Projekt auch ein sprachlich-kulturelles Projekt der ,Hebräisierung' war, das zudem die Vorstellung einer wehrhaften Männlichkeit transportierte: Der (männliche) Neue Jude sollte Hebräisch sprechen. Erst seitdem die Multikulturalität der israelischen Gesellschaft zur Debatte steht, werden Sprachkontakte und Sprachenvielfalt neu thematisiert. Denn trotz stark ideologisierter Zwänge erwiesen sich sprachliche Praktiken als resistent. Hier interessieren uns also jene Geschlechtervorstellungen, die innerhalb der Sprachbiographien mit verschiedenen Sprachen assoziiert werden. In diesem Sinne kann der Diskurs über eigenes Leben und Identität, der vorhandene Muster und historisch tradierte Vorstellungen in sich birgt, auch als eine Form des doing gender verstanden werden. Um dies genauer darzulegen, werden im Folgenden zunächst Äußerungen über die deutsche Sprache untersucht, die zwischen einer (männlich konnotierten) Gewalt-Sprache und einer gebildeten Kultursprache verortet sind. In einem zweiten Schritt wird der Druck, modernes Hebräisch zu sprechen, problematisiert. Abschließend sollen der Status der jiddischen Sprache sowie die Distinktionsstrategien, die Jeckes ihr gegenüber entwickelten, beleuchtet werden.

9 In der Oral-History-Forschung schätzt man, dass bereits ein Sample von 30 Interviews über das Individuelle hinaus exemplarischen und symptomatischen Charakter hat.

Io Vgl. Judith Butler, Das Unbehagen der Geschlechter, Frankfurt a. M. 1991; Susanne Beer, Marten Düring u. Elissa Mailänder, „Na Sie wissen ja, wie es mit den Männern so ist.“ Interaktive Geschlechterkonstruktionen in biografischen Interviews, in: Patrick Farges, Cécile Chamayou-Kuhn u. Perin Emel Yavuz Hg., Le lieu du genre. La narration comme espace performatif du genre, Paris 2011, 53-70. 


\section{Deutsch im Spannungsverhältnis zwischen Bildungssprache und Sprache der (männlichen) Gewalt}

Besonders emotionsgeladen war für die Jeckes in Palästina/Israel das Verhältnis zur deutschen Sprache. Der in Europa erlebte Antisemitismus wird nicht selten mit Erinnerungen an ein brutales, rohes und zeitgenössisch männlich konnotiertes Gebrüll, an eine brutale Befehlssprache narrativ verknüpft, dem gegenüber die schriftliche Sprache der großen (ebenfalls hauptsächlich männlichen) Repräsentanten deutscher Kultur steht. Symbolisch konnte diese gewaltvolle Übernahme der Sprache einer Form von Vergewaltigung gleichkommen - jedenfalls überwiegt dieses Gefühl etwa in den Erinnerungen des 1915 in Breslau geborenen Ephraim Orni: Er spricht von einer richtiggehend ,vergewaltigten Sprache', nachdem die Nazis ,in die deutsche Sprache hereingegangen" seien. ${ }^{11}$ Ähnlich äußert sich auch Jehoshua Arieli. Er wurde 1916 in Karlsbad geboren, kam bereits 1931 nach Palästina und diente im Zweiten Weltkrieg in der britischen Armee. Er verbrachte vier Jahre in deutscher Gefangenschaft. Besonders entsetzte ihn die brutal-männliche und sozial markierte Sprache der Unteroffiziere, die er im Gefangenenlager in Oberschlesien vernahm:

Wir haben sozusagen, wenn nicht mit dem allerschlechtesten, so doch mit dem zweit- oder drittschlechtesten Material zu tun gehabt. Besonders die Unteroffiziere waren das ganz verrohte Material gewesen. Und was mich so schrecklich abgestoßen hat, war ihr Deutsch. Das Deutsch, mein Deutsch, das ich im Ohr hatte, war das Prager Hochdeutsch, das man ruhig gesprochen hat. Aber die konnten nur schreien. Also erstens war das ein schreiendes Deutsch, und zweitens war das ein vollkommen primitives Soldatendeutsch, zum Teil Dialekt, ein schreiendes Soldatendeutsch. Vielleicht war das untereinander anders, aber wir haben sie ja nur als Herren gekannt. Vom Akustischen wie vom Inhaltlichen her war das ein Befehlsdeutsch. Und so haben wir das Deutsch nur in abschreckendster Art kennengelernt. [...] Wenn aber das Deutsche eine Gelehrtensprache ist, dann ist es etwas Anderes. ${ }^{12}$

II Israel-Korpus, 1. Generation (IK1), Interview Anne Betten mit Ephraim Orni (ehem. Fritz Schwarzbaum), Jerusalem, 20.4.1991; vgl. auch IK1, Interview Anne Betten mit Jehuda Steinbach, Tel Aviv, 24.4.1991. Bereits Victor Klemperer hatte in seiner sprachphilosophischen Beobachtung der Lingua Tertii Imperii (LTI - Notizbuch eines Philologen, Berlin 1947) das Phänomen der Sprachbrutalisierung beobachtet. Das Buch steht unter dem Motto „Sprache ist mehr als Blut“, nach einer Aussage Franz Rosenzweigs. Es beginnt statt eines Vorworts mit dem Kapitel „Heroismus“, in dem sich der Autor gegen die übertriebene Verwendung der Begriffe „Heldentum“ und „heldenhaft“ im Nationalsozialismus wendet. Klemperer kam zum Ergebnis, dass die NS-Sprache die Menschen weniger durch Reden und Flugblätter als durch die Häufung und Rekurrenz bestimmter, mit nationalsozialistischen Vorstellungen besetzter Begriffe beeinflusst habe. 
Hier wird das „verrohte“, „primitive Soldatendeutsch“ der „Gelehrtensprache“ gegenübergestellt. Der erzwungene Sprachkontakt innerhalb des Gefangenenlagers entblößt so eine soziale und kulturelle Hierarchisierung männlicher Verhaltensweisen, in der die deutschen Unteroffiziere akustisch eine rohe männliche Gewalt zur Schau stellen, während der Erzähler, ein deutschsprachiger Jude aus Böhmen, durch seine Sprache eine andere, bildungsbürgerliche, ,ruhige' und weiche Männlichkeit verkörpert.

Der Nationsbildungsprozess im deutschsprachigen Raum sowie der eng mit ihm verknüpfte Emanzipations- und Verbürgerlichungsprozess der Juden bergen wichtige geschlechtergeschichtliche Aspekte. Einerseits kristallisierten sich im Zuge der Nationsbildung spezifische Geschlechterrollen heraus: So wurde beispielsweise ein hegemoniales Modell ${ }^{13}$, deutscher ${ }^{6}$ Männlichkeit zunehmend mit soldatischen Werten, Wehrhaftigkeit sowie ,deutscher Ehre und Treue ${ }^{6}$ assoziiert. ${ }^{14}$ Andererseits entwickelten sich zeitgleich bildungsbürgerliche Lebenswelten, ${ }^{15}$ in denen sich ebenfalls Familien- und Geschlechterrollen herausbildeten: Auch hier entstanden Werte und Normen (bildungs)bürgerlicher Männlichkeit. Im späten 19. Jahrhundert entwickelten sich dann antisemitische Stigmatisierungen, die das vermeintliche Anderssein der Juden auf Körper- und Geschlechtsrepräsentationen verlagerten, Vorstellungen etwa von einer spezifisch jüdischen ,weichen', ,schwachen' beziehungsweise ,effeminierten'Männlichkeit. Dieser Form des Antisemitismus wurde mit Entwürfen einer (deutsch-)jüdischen Männlichkeit begegnet, die Aspekte des hegemonialen Modells der Wehrhaftigkeit adaptierten. Dazu gehörte etwa die Übernahme des populärkulturell und sozialdarwinistisch geprägten Begriffs des „Muskeljudentums “16, der Teil des Regenerierungsdiskurses des Zionismus wurde. Auch die Gendervorstellungen der nach Palästina Ausgewanderten schienen deutlich von diesem komplexen Spannungsverhältnis geprägt.

Den Antisemitismus in ihrer Jugend erlebten die deutschsprachigen Juden auch als körperliche und sprachliche Ausgrenzung durch Formen des hate speech. ${ }^{17}$ Junge Män-

I3 Laut R. W. Connell (Der gemachte Mann. Konstruktion und Krise von Männlichkeiten, Wiesbaden $2006^{3}$, 97) bilden moderne liberale Gesellschaften stets eine „hegemoniale Männlichkeit“ heraus, also ein bestimmendes Wertegefüge für Männer. Dem ist entgegengehalten worden, dass selten eine hegemoniale Männlichkeit für eine gesamte Gesellschaft verbindlich ist, sondern dass verschiedene soziale Gruppen sich an unterschiedlichen Männlichkeitskonstruktionen orientieren, vgl. Michael Meuser u. Sylka Scholz, Hegemoniale Männlichkeit. Versuch einer Begriffsklärung aus soziologischer Perspektive, in: Martin Dinges Hg., Männer - Macht - Körper. Hegemoniale Männlichkeiten vom Mittelalter bis heute, Frankfurt a. M. 2005, 211-228.

I4 Zum Modell deutscher Männlichkeit vgl. George L. Mosse, Das Bild des Mannes. Zur Konstruktion der modernen Männlichkeit, Frankfurt a. M. 1997; Ute Frevert, Die kasernierte Nation. Militärdienst und Zivilgesellschaft in Deutschland, München 2001.

Is Vgl. Andreas Gotzmann, Rainer Liedtke u. Till van Rahden Hg., Juden, Bürger, Deutsche. Zur Geschichte von Vielfalt und Differenz 1800-1933, Tübingen 2001.

I6 U.a. durch Max Nordau, der diesen Begriff in seiner Ansprache vor dem Zweiten Zionistenkongress (1898) gebrauchte.

17 Vgl. Judith Butler, Excitable Speech. A Politics of the Performative, London/New York 1997. 
ner wurden von anderen gemieden und nicht mehr als wehrfähige Körper wahrgenommen. Sie wurden nicht mehr angesprochen, sondern angebellt. Dies wird von Moshe (Max) Ballhorn (geb. 1913 in Berlin) körperbezogen erinnert, als er im Interview gerade seine Beziehung zur deutschen Sprache erwähnt:

Da ist wieder der Unterschied zwischen uns und unseren Kindern. [...] Die haben das Deutschtum nicht mit Löffeln gefressen, verstehen Sie, und nachher ein Tritt in den Hintern gekriegt. Die haben das niemals erlebt. Die haben niemals dieses tiefe seelische Erlebnis gehabt, ausgestoßen zu werden, rausgeschmissen zu werden aus ihrer Sprache und Heimat. ${ }^{18}$

Die antisemitische Ausgrenzung, das ,Ausgestoßenwerden', ist hier eindeutig noch nicht verdaut: Das damals zutiefst gekränkte Selbstverständnis Ballhorns als deutscher Mann wurde mit dem demütigenden „Tritt in [die Tabuzone des] Hintern“ nachhaltig gestört.

Bis heute ist das Verhältnis der israelischen Öffentlichkeit zur deutschen Sprache kein leichtes, auch wenn sich dies in letzter Zeit verändert hat und Deutsch gerade ,in ist. Aber in den 1950er Jahren war die ,Sprache der Mörder' aus dem öffentlichen Leben verbannt. In seinen Kindheitserinnerungen beschreibt Amos Oz das (besonders auf der Leinwand tradierte) stereotype Bild der deutschen Sprache. Nicht selten wurde die deutsche Sprache - sehr zum Bedauern der Jeckes - auf wenige von männlichen Soldaten gebrüllte Wörter (Befehle, Schimpfwörter oder technische Begriffe) reduziert:

Aus dem Mund eiskalter, bösartiger Nazis in Wehrmachtsuniformen oder in schwarzen SS- und Gestapo-Uniformen hörten wir dann, wieder und wieder, jenes Dutzend gebellter deutscher Wörter, bei denen es mir heute noch kalt über den Rücken läuft: raus, schnell, Achtung, kaputt, Zug, Eisenbahn, halt! und jawohl. ${ }^{19}$

Doch die Ablehnung gegenüber der deutschen Sprache war ambivalent. Zwar wurde die Sprache der Nazis verbannt, aber Deutsch war eben auch die Sprache der gebildeten Jeckes, die ihre Arroganz gegenüber der ,primitiven' Kultur des Jischuw bisweilen deutlich zum Ausdruck brachten. Die israelische Germanistin Na’ama Sheffi schreibt hierzu:

The anti-German attitude, which advocated a total boycott of all German imports, cultural or otherwise, was to some extent shared by the mainstream. The mainstream's attitude, however, was conditioned not only by hatred of Nazi Germany, but also by domestic problems with German-speaking immigrants. In the

I8 IK1, Interview Anne Betten mit Moshe (ehem. Max) Ballhorn, Tiberias, 1.7.1990.

I9 Amos Oz, Israel und Deutschland. Vierzig Jahre nach Aufnahme diplomatischer Beziehungen, Frankfurt a. M. 2005, 16. 
eyes of some of the Jewish population in Palestine, German-speakers suffered from two bad stigmas: One was the fact that they used the language of the greatest enemy the Jews had ever had. The other was the fact that many of them emigrated to Palestine not as committed Zionists, but simply refugees with no great ambition to integrate the Palestinian society. This elicited deeply malicious responses by some members of the intellectual elite. ${ }^{20}$

Trotz offizieller Verbannung wurden die Sprache sowie ,deutsche‘ Kulturschemata weitertradiert und es entstanden sprachliche Kontaktzonen im Bereich der Literatur (besonders der Kinderliteratur in hebräischer Übersetzung), der Alltagssprache und einiger Fachsprachen $^{21}$ (wie beispielsweise der technischen und der juristischen Sprache ${ }^{22}$ ) bis hin zum privaten jeckischen, ,muttersprachlichen' Familiengebrauch, dem jeckit meduberet oder jeckemed, mit dem intime Belange und Emotionen vermittelt wurden. ${ }^{23}$

Um den Stellenwert der deutschen Sprache in Palästina/Israel herauszuarbeiten, erscheint also eine epistemologische Vorgehensweise, die nach Konnotationen sucht, zielführender als eine statistische. ${ }^{24}$ Laut Dan Diner habe nämlich „die deutsche Sprache [...] für Juden eine Bedeutung, die in das Arsenal jüdischen Selbstverständnisses [gehört]. Schließlich war Deutsch unter Juden in Mittel- und Ostmitteleuropa im 19. Jahrhundert auch so etwas wie eine jüdische Sprache geworden. "25

Die Übersetzerin Aharon Appelfelds ins Deutsche, Anne Birkenhauer, hat durch ihre intensive Auseinandersetzung mit der Sprache des israelischen Schriftstellers eine solche

20 Na'ama Sheffi, Rejecting the Other's Culture - Hebrew and German in Israel 1933-1965, in: Tel Aviver Jahrbuch für Deutsche Geschichte, 27 (1998): Historische Migrationsforschung, hg. von Dan Diner, 301-320, 308. Vgl. auch Henry Wassermann, Das Deutsche in Erez Israel (1933-1948), in: Hermann Zabel Hg., Stimmen aus Jerusalem. Zur deutschen Sprache und Literatur in Palästina/Israel, Berlin 2006, 92-108.

2I Vgl. das von der Vereinigung der Israelis mitteleuropäischer Herkunft (Hebr. Irgun) herausgegebene Lexikon der Ben-Jehuda-Straße - ein Wörterbuch des gesprochenen Jeckisch in Israel [Hebr.], Tel Aviv 2012, das in Israel ein Bestseller ist.

22 Vgl. José Brunner, Deutsches Recht auf Hebräisch - Zur Entschädigung für NS-Verfolgte in Israel, Vortrag, internationale Tagung „Deutschland und Israel/Palästina von 1945 bis heute“, 15.-16. November 2012, Université Paris Ouest - Nanterre.

23 Dieser Stellenwert der deutschen Sprache wurde in den letzten Jahren gewürdigt. Anlässlich eines Tel Aviver Symposiums zum Thema „Deutsch in Israel“ (2012) erinnerte der ehemalige Vorsitzende der Gesellschaft für deutsche Sprache, Rudolf Hoberg, daran, dass ,auch nach Auschwitz für viele Juden Deutsch ihre Muttersprache war und ist“. Rudolf Hoberg, Deutsch in Israel - ein besonderes, schwieriges Thema, in: Der Sprachdienst, 57, 4-5 (2013), wie Anm. 4, 143-145, 144.

24 Vgl. Karin Neuburger, Die Geschichte der deutschen Sprache in Palästina/Israel, in: Der Sprachdienst, 57, 4-5 (2013), wie Anm. 4, 154-167.

25 Dan Diner, Jeckes - Ursprung und Wandel einer Zuschreibung, in: Moshe Zimmermann u. Yotam Hotam Hg., Zweimal Heimat. Die Jeckes zwischen Mitteleuropa und Nahost, Frankfurt a. M. 2005, 100-103, 101. Vgl. auch Stephan Braese, Eine europäische Sprache. Deutsche Sprachkultur von Juden 1760-1930, Göttingen 2010. 
Methode der „Spurensuche“ angewendet. Deutsch ist die Muttersprache Appelfelds, der in Czernowitz (Bukowina) geboren wurde: „Es ist die Sprache der frühen Kindheit, der Erinnerungen an die Familie. Deutsch ist die erste Sprache eines Menschen, der im Hebräischen zum Dichter wurde“, schreibt sie. Deutsch sei gleichzeitig „die Sprache seiner Mutter und die Sprache der Mörder seiner Mutter“. ${ }^{26}$ Somit sei auch seine tief verankerte „Muttersprache“ getötet worden, die sich wie ein Geist immer wieder manifestiere: „Irgendwo spukt durch Appelfelds hebräische Texte immer wieder die deutsche Muttersprache, so ähnlich vielleicht wie die Geister, die in so vielen seiner Bücher erscheinen ". ${ }^{27}$

Für viele Jeckes war die Suche nach den Wurzeln und Spuren einer anderen, nichtsoldatischen deutschen Sprachtradition von besonderer existentieller und identitätsstiftender Bedeutung. Oft sind Aussagen über Reminiszenzen und Remanenzen der deutschen Sprache eng mit persönlichen Identitätsgefühlen, ja mit intimer Geschlechtsidentität verknüpft. Im Interview von Anne Betten mit Moshe Ballhorn kommt zum Ausdruck, dass er sich nur deswegen von den Nazis nicht habe ,entmannen“ lassen, weil er seine eigene - die deutsche - Sprache beibehalten habe: „Die deutsche Sprache ist mein persönliches Eigentum, die kein Hitler mir wegnehmen konnte mit seinen deutschen Männern und seinen deutschen Frauen. "28 Geschlechteridentität, persönliche Identität und Zugehörigkeitsgefühl zur deutschen Kulturnation sind hier narrativ eng miteinander verwoben. Im Auto-Interview äußert der 1923 in Wien geborene Joseph Amit eine ähnliche intime Verbundenheit mit der Sprache, indem er die Geschichte eines gewissen „Dr. Warburg“, eines typischen Jeckes, erzählt:

Es gab in Kirjat Anavim den Doktor Warburg - ein kleiner Arzt, ein Dorfdoktor, von einer berühmten Familie stammend, die in der Finanz war. Aber er hat sich dazu wahrscheinlich nicht geeignet und er verließ seine Familie und versuchte sein Leben allein als kleiner Dorfarzt aufzubauen. Aber er hat sich eigentlich nie wieder eingelebt. Immer wenn er Hebräisch sprach, sprach er so wie ein junger Bursche oder ein bisschen kindisch. Und im Augenblick, wo er Deutsch sprach, hat seine Kultur geglänzt! ${ }^{29}$

Hier werden sozialer und geschlechtsspezifischer Status direkt mit der sprachlichen Ausdrucksfähigkeit verbunden. Wer sich nicht ausdrücken kann, ist kein ganzer Mann, sondern bleibt zeitlebens ein „junger Bursche oder ein bisschen kindisch“.

26 Anne Birkenhauer, Die Suche nach dem richtigen Wort: Probleme beim Übersetzen von Aharon Appelfeld ins Deutsche, in: Gisela Dachs Hg., Sprachen (Jüdischer Almanach des Leo Baeck Instituts), Frankfurt a. M. 2007, 43-55, 44.

27 Birkenhauer, Suche, wie Anm. 26, 46f.

28 IK1, Interview Anne Betten mit Moshe (ehem. Max) Ballhorn, Tiberias, 1.7.1990. 


\section{Neuer Jude, sprich Hebräisch!}

Der identitäre und intime Aspekt der Sprache in den Selbstzeugnissen lässt darauf schließen, dass im post-migratorischen Prozess starke Zwänge ausgeübt wurden, denen gegenüber diejenigen, die in der zionistischen Geschichtsschreibung als Mitglieder der ,Fünften Alija' galten, Remanenzstrategien einsetzten. Die Beziehung der deutschen Juden und Jüdinnen - gerade auch der deutschen ZionistInnen ${ }^{30}$ - zur neu-hebräischen Kultursprache ist äußerst ambivalent. Die drei Säulen des Zionismus waren die Errichtung eines jüdischen Staates, die Schaffung einer Nationalsprache auf Grundlage einer wiederbelebten Kultursprache und die Vorstellung eines Neuen Juden, der diese Regenerierung vollenden und verkörpern sollte. Der Staat Israel baute nach 1948 auf der Negation der europäischen Kultur- und Sprachtraditionen der Galut (Diaspora) auf, und das wiederbelebte Hebräisch wurde zur Nationalsprache. Besonders angeprangert wurde die „schwärzeste aller Exil-Existenzen“"31: die deutsch-jüdische Tradition. Dabei ahmte der Zionismus paradoxerweise viele Aspekte anderer Nationalbewegungen nach, besonders der deutschen Nationalbewegung. ${ }^{32}$ Angesichts dieses Widerspruchs ist es nicht verwunderlich, dass in den Aussagen der Jeckes eine gewisse Distanzierung gegenüber dem zionistischen Imperativ des Daber Iwrit - „Sprich Hebräisch“ - zu hören ist.

Die deutsch-jüdischen Einwanderer der 1930er Jahre erreichten Palästina zu einem Zeitpunkt, als Neu-Hebräisch sich bereits als Kommunikationssprache etabliert hatte. ${ }^{33}$ Doch in vielerlei Hinsicht entsprachen sie nicht dem Ideal des Neuen Juden und wehrten sich, es zu verkörpern. Der Hebräisierungsprozess war mit starken Zwängen verbunden. Im Interview erinnert sich Paul Alsberg (geb. 1919 in Wuppertal) an den Druck, Hebräisch zu sprechen, als ein regelrechtes Gefecht, und viele Jeckes seien in

30 Innerhalb des deutschsprachigen Zionismus war die Frage der Sprache, die man in Erez Israel sprechen solle, eine besonders heikle. Vgl. Moshe Zimmermann, Die deutschen Juden, 1914-1945, München 1997, 106ff.

3I Michael Brenner, Symbiose oder Selbsttäuschung. Rückblicke auf das deutsche Judentum, in: Nicolas Berg, Omar Kamil, Markus Kirchhoff u. Susanne Zepp Hg., Konstellationen. Über Geschichte, Erfahrung und Erkenntnis. Festschrift für Dan Diner zum 65. Geburtstag, Göttingen 2011, 143-156, 155.

32 Schlüsseltexte des Zionismus wurden in deutscher Sprache verfasst. Olivier Baisez meint: „Selbst wenn deutsche Zionisten selbstverständlich eine jüdische Identität in Anspruch nahmen, verstanden sie sich stets auch als deutsche Komponente einer weltweiten Bewegung und sprachen von Deutschland aus. In den letzten Jahren des 19. Jahrhunderts und bis zum Ende des Ersten Weltkriegs wurde ein wesentlicher Teil des zionistischen Diskurses über die jüdische Kolonisation Palästinas in deutscher Sprache und im Deutschen Kaiserreich veröffentlicht, häufig von Persönlichkeiten, die an deutschen Hochschulen ihre Ausbildung absolviert hatten." Olivier Baisez, Zentralität in den Kolonisationsplänen der deutschen Zionisten. Vom ersten Zionistenkongress (1897) bis zum Ende des Ersten Weltkriegs, in: Germanistik in der Schweiz, 10 (2013), 13-20, 13.

33 Miryam Du-nour, Modernes Hebräisch - die vorherrschende Sprache innerhalb der jüdischen Gemeinschaft in Palästina, in: Betten/Du-nour, Sprachbewahrung, wie Anm. 4, Teil II (Phonai 45), $210-216$. 
dieser Hinsicht die Verlierer eines Sprachkampfes gewesen. ${ }^{34}$ Michael Dak, Schriftsteller, Kritiker, Intellektueller und Angehöriger der zweiten Jeckes-Generation, schreibt dazu: „Und das Hebräische? Ein Teil der Einwanderer wandte sich von der neuen Sprache ab. Iwrit saffa kascha, Iwrit schwere Sprache, sagten sie, der deutschen Syntax entsprechend. Die fremden Laute trafen auf zusammengepresste Lippen. " ${ }^{35}$

Außerdem veränderte sich im Rahmen des israelischen Nationsbildungsprozesses und des quasi-permanenten Kriegszustandes die Sprache selbst. Hebräisch wurde in Wortschatz, Syntax und Tonfall zunehmend zur männlich-virilen Sprache. Doch gerade die Jeckes erinnerte das maskulinisierte Hebräisch an die Brutalisierung der deutschen Sprache während der Nazizeit. Ironisch meint Abraham Goldberg (geb. 1923 in Altenburg) hierzu: „Wenn ich mit jemandem schimpfen will, dann schrei ich auf Iwrit. Das ist mir viel bequemer. “36 Als Jeckes-Sohn hat Michael Dak ein besonderes Gespür für jene Entwicklung, die aus Hebräisch die „Sprache von Hightech, Landwirtschaft und Industrie, von Soldaten und Kontrollpunkten“ - allesamt Avatare des Neuen Juden - machte. Er bedauert, dass Hebräisch so effizient geworden sei und demnach voll von „vorzugsweise einsilbigen Wörtern, damit auch die sehr Beschäftigten Zeit finden, sie fachmännisch auf der Zunge zu rollen“. Er klagt darüber, dass „diese meine Sprache, die so männlich geworden ist, kaum egalitäre Züge aufweist, ständig die maskuline Verbform verwen-

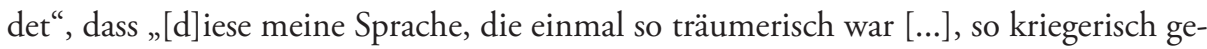
worden ist". ${ }^{37}$ Abraham Kadimah (geb. 1925 in Wien) hält seinerseits fest, die hebräische Sprache sei im Vokabular - und folglich in der Semantik - seit der Gründung Israels vereinfacht worden, um eine brutale Effizienz zu erreichen: „Diese Sprache wird hier verschandelt“, meint er im Interview. ${ }^{38}$ Auch Abraham Eran (geb. 1907 in Stettin) sieht im gesprochenen Hebräisch eine arme, „unterentwickelte “ Sprache, die sich lediglich dazu eigne, technische und logistische Aspekte auszudrücken. ${ }^{39}$ Diese Entwicklung erlebten die Jeckes mit und sie bemühten sich zuweilen, den Rhythmus des gelehrten Sprechers - mit starkem deutschen Akzent - zu bewahren.

Der Druck kam auch von der Umkehrung der Familienschemata, weil das Erlernen der neuen Sprache (Hebräisch) zu neuen Rollenverteilungen führte: „Hebräisch ist die einzige Muttersprache, die die Mütter von den Kindern lernen“, ${ }^{40}$ meint Else Admoni

34 IK1, Interview Anne Betten mit Paul (und Betti) Alsberg, Jerusalem, 25.4.1994.

35 Michael Dak, Israelis aus Not, in: MB Yakinton. Mitteilungsblatt der Vereinigung der Israelis mitteleuropäischer Herkunft, 2007, unter: http://www.irgun-jeckes.org/?CategoryID=254\&Articl eID=211, Zugriff: 20.6.2014.

36 IK1, Interview Kristine Hecker mit Abraham (ehem. Adolf) Goldberg, Ramat Gan, 4.7.1990.

37 Michael Dak, Deutsch und Hebräisch - Die Geschichte einer Hassliebe, aus dem Hebräischen übersetzt v. Ruth Achlama, in: Dachs, Sprachen, wie Anm. 26, 20-26, 22.

38 IK1, Interview Eva Eylon mit Abraham Kadimah (ehem. Walter Metzer Ruhig), Ramat Gan, 18.8.1991.

39 IK1, Interview Eva Eylon mit Abraham Eran (ehem. Otto Ehrenwerth), Jerusalem, 22.6.1992. 
(geb. 1908 in Fulda). Auch der 1906 in Luckenwalde geborene Mordechai Heinz Gilead, der zur älteren Jeckes-Generation gehört, sagt im Interview, er habe damit leben müssen, dass ihn seine Kinder immer wieder im Hebräischen korrigierten, sodass sein traditionelles Verständnis der Vaterrolle dadurch verändert worden sei. ${ }^{41}$

Ein besonders komplexes Verhältnis zur hebräischen Sprache hat der 1932 in Czernowitz geborene Aharon Appelfeld entwickelt, ein Verhältnis, das außerdem von seiner bereits erwähnten gestörten Beziehung zur deutschen (Mutter-)Sprache bestimmt wird. Der Querdenker Appelfeld ist dafür bekannt, dass er sich in seinen Romanen konsequent von zwei in der israelischen Literatur dominierenden Themen entferne: In den kollektivistischen Aufbaujahren Israels erzählte er nicht vom pionierhaften Heldentum der Hebräer und der Neuen Juden, sondern von der Situation der Juden in der Galut (Diaspora); außerdem kommt in den meisten seiner Bücher die Shoah selbst nicht vor, sondern sie schwebt vielmehr als Schatten über seinen Erzählungen. Im Dokumentarfilm „D’une langue à l'autre“ (2004) wird Appelfeld von der Regisseurin Nurit Aviv zum Thema „Sprache und Sprachkontakte“ interviewt. Nach dem Krieg lernte Appelfeld Hebräisch. Diesen Lernprozess schildert er als mit Zwängen und Imperativen verbunden:

Nach und nach lernten wir Hebräisch. Es war mir eine große Mühe, diese Sprache, die so anders ausgesprochen wurde als die Sprachen, die ich kannte, zu vereinnahmen. Sie klang wie Befehle: Gehen! Schlafen! Aufräumen! [...] Der Einwanderer wurde 1946, d. h. in den 1940er und 1950er Jahren, nicht akzeptiert. Das Land war ideologisch und die Ideologie befahl: Daber Iwrit (Sprich Hebräisch)! Vergiss, vergiss Deine Vergangenheit, vergiss Deine Muttersprache, vergiss, wer Du warst. ${ }^{42}$

Die Übersetzerin Appelfelds, Anne Birkenhauer, schreibt, man merke Appelfelds geschriebenem Hebräisch etwas Fremdes an. Sein Hebräisch verliere an Selbstverständlichkeit: „Es ist seine Art der Entautomatisierung des Hebräischen “43 und eine Distanzierung gegenüber dem gängigen, maskulinisierten und technisch-soldatischen Hebräisch. Auch klinge Appelfelds gesprochenes Hebräisch - so Birkenhauer - zart und weiblich, alles andere als maskulin.

4I IK1, Interview Kristine Hecker mit Mordechai Heinz Gilead (ehem. Heinz Guttfeld), Ramat Gan, 4.7.1990.

42 Interview Aharon Appelfeld (deutsche Untertitel), in: Nurit Aviv, D'une langue à l'autre, Dokumentarfilm, Swan Production/ZDF/Transfax Films/Noga Channel 8/Dérives, 2004, 55 Min. (DVD: Éditions Montparnasse 2011).

43 Birkenhauer, Suche, wie Anm. 26, 47. 


\section{4. ,Jiddischkayt' versus, Jeckischkeit'}

Als Resistenzstrategie zu den (Gender-)Zwängen im jungen Israel habe sich Appelfeld dazu entschlossen, Jiddisch, die Sprache der Großeltern und die weiblich konnotierte ,Mameloschen' (Muttersprache), wiederzubeleben:

Und so lernte ich Jiddisch. Ich lernte Jiddisch, um Deutsch zu verjagen. Da gibt es sicher sehr komplexe psychologische Motivationen. Ich stamme aus einer assimilierten Familie und jede assimilierte Familie verdrängte ihr Jüdischsein. Und Jiddisch war das Symbol dieses Jüdischseins. Um also alles Jüdische zu erfahren, lernte ich Jiddisch. Ich kann Jiddisch, aber nicht perfekt. Ich kann es lesen und schreiben. Ich lese jiddische Literatur und jiddische Essays. Diese Sprache liegt mir am Herzen, denn sie war die Sprache meiner Großeltern. Auch habe ich den Tod mit jiddischen Augen gesehen. Ich sah alte Juden, schwache Frauen, Kinder an der Schwelle des Todes und alle sprachen Jiddisch. ${ }^{44}$

Gerade Jiddisch, das zugleich Deutsch und Nicht-Deutsch ist, gilt Aharon Appelfeld als Resistenzstrategie. Das Wiederbeleben der jiddischen Sprache, das zugleich auch ein Erlernen ist, hilft ihm, die eigene deutsche Muttersprache zu verdrängen.

Auch für die Jeckes spielte sich im Kontakt mit Jiddisch eine historische und kulturelle Konfrontation mit dem ,Ostjudentum' ab. Stellvertretend hierfür stehen kann die Aussage von Amoz $\mathrm{Oz}$ in seiner Autobiographie: „Auf der Werteskala meiner Eltern galt: je westlicher, desto kultivierter". ${ }^{45}$ Die Ankunftsgesellschaft der Jeckes - die jüdische Gemeinschaft im Mandatsgebiet Palästina oder Jischuw ${ }^{46}$ - war mehrheitlich von osteuropäischen (besonders russischen) jiddischsprachigen Juden geprägt. Und sogar die ,Fünfte' (sogenannte ,deutsche') Alija war nicht mehrheitlich deutschsprachig: Die meisten Einwanderer stammten gar nicht aus Deutschland, Österreich oder der Tschechoslowakei. Unter den rund 280.000 Einwanderern der Jahre 1933 bis 1939 machten Deutschsprachige nur etwa ein Fünftel aus. ,Jeckischkeit' war also kein fester Begriff, vielmehr musste sie immer wieder unter Beweis gestellt werden, gerade in der Auseinandersetzung mit dem Jiddischen. So lässt sich auch erklären, warum ,echte` Jeckisch-

44 Interview Aharon Appelfeld, wie Anm. 42. Vgl. Masha Itzhaki, Le yiddish: un passé proche et un souvenir éternel dans l'univers d'Aharon Appelfeld, in: Yod. Revue des études hébraïques et juives, 16 (2011), hg. von Yitskhok Niborski, 51-59.

45 Amoz Oz, Eine Geschichte von Liebe und Finsternis. Übersetzt v. Ruth Achlama, Frankfurt a. M. 2004, 8. Zum Phänomen der jüdischen „Westverschiebung“ schreibt Dan Diner: „[D]ie Geschichte der Juden [war] von jeher mit den Phänomenen der Verschiebung europäischer Lebenswelten auf der kulturgeographischen Ost-West-Achse thematisch vertraut gewesen.“, in: ders., Gedächtniszeiten. Über jüdische und andere Geschichten, München 2003, $12 \mathrm{f}$.

46 Vor den 1930er Jahren stellte der Jischuw etwa 18 Prozent der Bevölkerung Palästinas dar, die sich insgesamt auf 1.080.000 BewohnerInnen belief. 
keit so umstritten war (und ist). Gabriel Alexander erinnert an jene „feinen Unterschiede“, die „waschechte Jeckes“ oder „koschere Jeckes “ ${ }^{\text {“47 }}$ machten, wenn sie einen als Jecke ,getarnten“ (jiddischsprachigen) Ostjuden entlarvten: „Die Einwohner von Reha$v^{4} a^{48}$, zu denen auch meine Eltern gehörten, betrachteten sich als echte Jeckes, da ihre Familien über viele Generationen in Deutschland oder Österreich ansässig gewesen waren, und hatten für die falschen Jeckes nur Sarkasmus übrig. " ${ }^{49}$

Bei weitem nicht alle deutschsprachigen Juden und Jüdinnen, die in den 1930er Jahren in Palästina einwanderten, waren also aus Deutschland oder Österreich gebürtig oder sie waren es im Hinblick auf die eigene Familiengeschichte nicht lange gewesen. ${ }^{50}$ Vielmehr hätten sich die Jeckes die Fünfte Alija „erst rückwirkend angeeignet“, 51 so Gabriel Alexander weiter. Dies sei, so Dan Diner, nur möglich, seitdem es in Israel legitim ist, „die jeweiligen Vorgeschichten der verschiedenen Judenheiten herauszustellen“, seitdem „ein homogener Israelismus“ nicht mehr allgemeines Ideal ist. ${ }^{52}$

Der Sprach- und Kulturkontakt mit jiddischsprachigen Juden aus Osteuropa prägte demnach die Jeckes in Palästina/Israel. Den meisten lag besonders daran zu zeigen, dass im Elternhaus ,gutes Deutsch' gesprochen wurde. Dies wurde auch (und gerade) von jenen betont, deren Eltern aus Osteuropa nach Deutschland oder Österreich ausgewandert waren. ${ }^{53}$,Gutes Deutsch ' bedeutete natürlich vor allem ,nicht Jiddisch', aber auch ,dialektfrei‘. Dies erklärt den hohen Stellenwert des Hochdeutschen. Der Sprachwissenschaftler Jacob Toury meint hierzu: „Ein deutscher Professor konnte sächseln, berlinern, schwäbeln, wienern oder Goethes Frankfurterisch sprechen. Ein gebildeter Jude sprach Hochdeutsch. Das war seine Auszeichnung, das war aber auch sein Manko." 54

47 Micha Limor, Was ich noch sagen wollte: Die Jeckes-Identität, in: MB Yakinton. Mitteilungsblatt der Vereinigung der Israelis mitteleuropäischer Herkunft, 79, 246 (2011), unter: http://www.irgun-jeckes.org/?CategoryID=451\&ArticleID=2453, Zugriff 13.2.2015.

48 Rehavia ist ein vornehmes Viertel in West-Jerusalem.

49 Gabriel Alexander, Jeckes im Buch Taschach (1948), in: MB Yakinton. Mitteilungsblatt der Vereinigung der Israelis mitteleuropäischer Herkunft, 80, 251 (2012), unter: http://www.irgun-jeckes.org/? CategoryID=483\&ArticleID=2641, Zugriff: 24.6.2013. Vgl. auch Friedrich Reichenstein, Yedioth Hayom - Ich und meine Zeitung, eine erlebte Geschichte, Tel Aviv 2007, $12 \mathrm{f}$.

50 Vgl. Gideon Greif, Colin McPherson u. Laurence Weinbaum, Vorwort, in: dies., Die Jeckes. Deutsche Juden aus Israel erzählen, Köln/Weimar/Wien 2000, X.

5I Vgl. Gabriel Alexander, Leserbrief, in: MB Yakinton. Mitteilungsblatt der Vereinigung der Israelis mitteleuropäischer Herkunft, 80, 256 (2012), 5.

52 Dan Diner, Geleitwort, in: Greif/McPherson/Weinbaum, Jeckes, wie Anm. 50, VII-VIII, VIII.

53 So Anne Betten, Vielleicht sind wir wirklich die einzigen Erben der Weimarer Kultur, in: dies./Dunour, Sprachbewahrung, wie Anm. 4, Teil II (Phonai 45), 157-181, 174.

54 Jacob Toury, Die Sprache als Problem der jüdischen Einordnung im deutschen Kulturraum, in: Jahrbuch des Instituts für Deutsche Geschichte (Tel Aviv), 11 (1982): Gegenseitige Einflüsse deutscher und jüdischer Kultur, hg. von Walter Grab, 75-96, 84. 
Auch die Schriftstellerin und Übersetzerin Ada Brodsky beschreibt ihr Deutsch als „ein akzentfreies Deutsch, [...] das nirgendswohin (sic) gehört“. 55 Zudem vollzog sich die Abgrenzung gegenüber Jiddisch über geschlechterspezifische Zuweisungen. Nur selten würdigten Jeckes das Jiddische, meist nahmen sie es lediglich als kindisches beziehungsweise verweiblichtes Randphänomen wahr, als ,Mameloschen' oder ,WeiberTeitsch' - so die historischen Bezeichnungen des Jiddischen. Die in Deutschland geborene Mutter des israelischen Historikers Tom Segev hörte nur deshalb den jiddischen Rundfunk in Israel, weil sie sich über die Sprache lustig machen konnte. ${ }^{56}$ In seiner Autobiographie - betitelt „Die Jeckes sind immer marschiert“ - zitiert der 1920 in Neubiberg geborene Arie Nir (ehem. Leo Blumenfeld) Bruchstücke eines kindischen Kauderwelsches, das er als Jiddisch bezeichnet: „Wir sinnen gesessen bei di Quallen [Quellen] und die Beduina sinnen gekimmen mit die Steckschiach [Pantoffeln]. "

Die ,Mameloschen' war in Deutschland schon vor der Emigration verachtet worden und den Jiddischsprachigen war der (männlich konnotierte) Kern der deutschen Kulturnation verwehrt geblieben. So lässt sich vielleicht auch Aharon Appelfelds Entscheidung, Jiddisch zu lernen, deuten. Außerdem wehrt er sich damit auch gegen den männlichen Hegemonialanspruch des Hebräischen in Israel und kann sich mit Jiddisch als einer intimen, weiblichen Sprache identifizieren, mit einer Sprache also, die außerhalb des nationalen Paradigmas steht, die weder „Armee noch Flotte“ besitzt, um das Max Weinreich zugeschriebene Bonmot zu paraphrasieren. ${ }^{58}$ Auch der israelische Journalist, Schriftsteller und Jiddischist Benny Mer behauptet dies:

Die Neuentdeckung des Jiddischen ist hier [in Israel] oft Ausdruck des Protests gegen den hegemonialen Anspruch des Hebräischen und der zionistischen Ideologie, gegen die kulturelle, ja vielleicht auch politische und gesellschaftliche Eindimensionalität im Land. In der lange von Werten der physischen Stärke, der Männlichkeit und der Militanz geprägten israelischen Gesellschaft scheint gerade mameloschen, die jiddische Muttersprache, die ganz ohne martialisches Vokabular auskommt, einen besonderen Reiz zu haben. ${ }^{59}$

55 IK1, Interview Miryam Du-nour mit Ada Brodsky (geb. Neumark), Jerusalem, 8.5.1991.

56 Vgl. Interview Patrick Farges mit Tom Segev, Jerusalem, 28.4.2013.

57 Arie Nir, Die Jeckes sind immer marschiert, in: Peter Zinke Hg., Flucht nach Palästina. Lebenswege Nürnberger Juden, Nürnberg 2003, 106-110, 109.

58 Manche Jiddischisten gehen noch weiter und unterstreichen das queere Potential von Jiddisch, so etwa Gilles Rozier. Vgl. die Beiträge der internationalen Tagung „Permanence du yiddish“ (Paris, 12.-13. November 2012), unter: http://permanence-du-yiddish.org/textes/conferenciers/ROZIERGilles-Allocution-Francais.pdf, Zugriff: 31.5.2013.

59 Benny Mer, Keine Sorge, die Sprache ist tot - Zum Gerücht über die Auferstehung des Jiddischen, aus dem Hebräischen übersetzt v. David Ajchenrand, in: Dachs, Sprachen, wie Anm. 26, 111-121, 119 f. 
Für die Jeckes aber war es unmöglich, diese Form der Andersheit in Anspruch zu nehmen: Zu groß war die sozio-kulturelle Distanz zur jiddischen Welt. Denn ,Jeckischkeit als kulturelles und symbolisches Kapital, das sich von der neu-hebräischen Kultur und von der jiddischen Tradition klar unterscheidet, erscheint in den Interviews als eine distinktive Identität innerhalb der israelischen Gesellschaft, die mit bestimmten Vorstellungen von Kultur und Geschlecht einhergeht.

Die ,Jeckischkeit' der eigenen Familie - das heißt das Beherrschen der deutschen Hochsprache, die Identifikation mit deutscher Bildung und Kultur sowie als ,deutsch identifizierte Körperhaltungen und Geschlechterperformanzen - konnten daher als Mittel der sozio-kulturellen Positionierung innerhalb der israelischen Gesellschaft eingesetzt werden, besonders von denjenigen, deren Jeckischkeit nicht selbstverständlich schien. Die Kulturwissenschaftlerin Rakefet Sela-Sheffy formuliert dies folgendermaßen: „It seems [...] that, despite their heterogeneity, a Yekke identity unified the German-speaking Jewish immigrants and made them a more cohesive cultural group than they were before immigrating. [...] Precisely because [those with an ostjüdisch past] were self-made Germans, they were reluctant to give it up [this German identity]. " ${ }^{0}$

\section{Schluss}

Die Jeckes haben sich in Israel integriert, viele sind aber gleichzeitig „eigen-sinnig“ geblieben. ${ }^{61}$ Ihre eigen-sinnige Art der Akkulturation war eine Form der „integration through distinction“. ${ }^{22}$ Jeckischkeit als „symbolic ethnicity“63 ist demnach als retrospektives Konstrukt der Post-Migration im Kontext von Sprach- und Kulturkontakten zu verstehen. Dies ermöglicht es den Jeckes, in den retrospektiven Selbstzeugnissen durchaus alternative Geschlechtsentwürfe zu formulieren, die mit dem zionistischen

60 Rakefet Sela-Sheffy, 'Europeans in the Levant' Revisited - German Jewish Immigrants in 1930s Palestine and the Question of Culture Retention, in: Tel Aviver Jahrbuch für deutsche Geschichte, 41 (2013): Deutsche(s) in Palästina und Israel. Alltag, Kultur, Politik, hg. von José Brunner, 40-59, 47 u. 50 .

6I Vgl. Katharina Hoba und Joachim Schlör, die sich auf den vom Alltagshistoriker Alf Lüdtke geprägten Begriff des „Eigen-Sinns“ berufen: dies., Die Jeckes - Emigration nach Palästina, Einwanderung ins Land Israel, in: Heimat und Exil. Emigration der deutschen Juden nach 1933 (Begleitbuch zur gleichnamigen Ausstellung im Jüdischen Museum Berlin), Frankfurt a. M. 2006, 103-110, 104. Zu den Distinktions- und Abgrenzungsstrategien vgl. Anne Betten, Die Akkulturation der deutschsprachigen Immigranten in Israel: Berichte aus heutiger Perspektive, in: Daniel Azuélos Hg., Alltag im Exil, Würzburg 2011, 59-78.

62 Rakefet Sela-Sheffy, Integration through Distinction: German Jewish Immigrants, the Legal Profession and Patterns of Bourgeois Culture in British-Ruled Jewish Palestine, in: Journal of Historical Sociology, 19, 1 (2006), 34-59.

63 Vgl. Herbert Gans, Symbolic Ethnicity: The Future of Ethnic Groups and Cultures in America, in: Ethnic and Racial Studies, 2, 1 (1979), 1-20. 
Programm des israelischen Nationalaufbaus nicht unbedingt übereinstimmten. ${ }^{64}$ Das konsequente Pflegen, ein ganzes Leben lang, einer grammatikalisch korrekten Hochsprache, jenes gute,Weimarer Deutsch'beziehungsweise ,Burgtheaterdeutsch', kann einerseits auf einen besonderen „Drill zur Norm“"65 in den Jugendjahren, andererseits aber auch auf sprachlich-emotionale Distinktionsstrategien zurückgeführt werden. Sprachlich wurde auf diese Weise eine andere deutsche Tradition bewahrt als die soldatisch-brutale. Diese deutsche Sprache wurde so zu einem Instrument, mit dem den maskulinistischen Tendenzen des Neu-Hebräischen beziehungsweise dem mit dem Jiddischen assoziierten Stigma der, Verweiblichung' widerstanden - und eine alternative Form israelischer Männlichkeit entwickelt werden konnte.

64 Vgl. hierzu Danny Kaplan, The Men We Loved. Male Friendship and Nationalism in Israeli Culture, New York/Oxford 2007.

65 In der Diskussion mit Anne Betten gibt Ephraim Orni zu, dass gerade dieser „Drill zur Norm“ mit den negativen Seiten der deutschen Tradition und Geschichte in Verbindung zu bringen sei. IK1, Interview Anne Betten mit Ephraim Orni (ehem. Fritz Schwarzbaum), Jerusalem, 20.4.1991. 